\section{LA UNIVERSIDAD FRENTE A \\ LAS NECESIDADES Y DEMANDAS \\ LOCALES /TERRITORIALES}

\author{
Silvina A. Sturniolo
}

La intención del presente artículo es problematizar el resurgimiento de las demandas por una atención a lo local/territorial/ o a la comunidad local que se realiza a la universidad, demandas que a su vez son acordes a los procesos de cambio social, y en las que subyace una discusión acerca de la redefinición de la función social de las universidades. El período 1995/2000 puede ser considerado como representativo de las tendencias de cambio de las decisiones en la creación de nuevas carreras y de diferentes consideraciones y servicios hacia lo local. Los discursos de presentación se configuran en torno a esta política como un rasgo diferencial, caracterizadas por un intento de acción o de relación hacia o con la comunidad. Surge en este contexto una demanda hacia una vinculación con el entorno, por la atención a lo local, por la necesidad de respuestas territoriales. Lo local es una de las formas discursivas presentes en el ámbito académico en la última década. Lo local como categoría nos remite al vinculo universidad- sociedad, ampliamente debatido redefinido y necesariamente cuestionado.

Palabras-Clave: Demandas. Función social. Universidad.
Licenciada en Ciencias de la Educación - Facultad de Filosofía y Letras - Universidad Nacional de Buenos Aires; Becaria doctoral CONICET, sede IICE/ FFyL - UBA; Docente FFyL y FCsSoc - UBA. Buenos Aires [Argentina] silvinasturniolo@hotmail.com 


\section{Múltiples demandas}

La formulación moderna de la universidad la concebía como lugar de producción y transmisión de conocimientos, y definía su función como institución universitaria de formar para responder a las necesidades y las demandas sociales. Hoy no sólo resulta impensable cuestionar esa función de formadora de cuadros profesionales acordes a las realidades nacionales y regionales, no obstante el debate actual interpela a la institución desde nuevas demandas acordes a los procesos de cambio social en las que subyace una discusión acerca de la definición de un vínculo local en oposición a lo global.

En los últimos años, se incorporan otros actores que se convierten en voces importantes para la definición de reformas y cambios en lo que respecta a la educación superior. Entre ellos encontramos organismos de crédito (BM, BID) Estado, gobernaciones o municipios, exigencias del mercado profesional, empresas multinacionales, nacionales, Pymes $^{\mathrm{I}}$, organizaciones sociales, entre otros. Tal como plantea F. Naistaht ${ }^{2}$ "[...] la relación de la universidad con su entorno experimenta una multiplicidad de niveles que es difícil reducir a los esquemas tradicionales en términos de estado y sociedades nacionales."

Hoy, acompañando la crisis endémica en la cual se encuentra nuestra institución, se hacen presentes series de demandas que la interpelan y plantean una exigencia de transformación. Demandas y exigencias provenientes de distintos sectores, actores y organismos nacionales o internacionales. Como resultado, la universidad deviene espacio de recepción de múltiples demandas, que no solo la interpelan sino que condicionan y comprometen su integridad como tal.

Desde muchas esferas (económicas, sociales, políticas) se considera que la universidad es una fuente muy importante para el desarrollo local del lugar donde se encuentra emplazada, hoy es interpelada por una mayor vinculación y aporte a la sociedad en la que se inscribe.

El retorno a lo local/ territorial y al énfasis en esa relación, trae aparejada varios supuestos y definiciones. En este sentido, es posible encontrar desde 
muchos autores y bibliografía internacional ${ }^{3}$ referida al tema, que el sentido principal se traduce en la importancia de la universidad para el desarrollo económico (cabe resaltar, que en algunos casos se intenta potenciar no sólo lo económico sino también lo social - local).

Se entiende que, si bien la universidad no es el único agente que pueda favorecer el desarrollo de su entorno

[...] debe aprender a interactuar con las instituciones locales y las empresas, aportando, además de formación e investigación, métodos, procedimientos y herramientas para analizar prospectivamente las necesidades del entorno local, para anticipar escenarios, y cooperar en la gestión del proyecto de futuro del territorio [...] (TOSTADO, 2004).

o bien como un "deber ser" para la universidad, si bien la misión clásica de la universidad no la define por la respuesta directa a problemáticas locales, la universidad puede y debe atender a las demandas de su entorno.

En este marco, subyace un enunciado que afirma que la universidad esta separada de la sociedad y ubica a la universidad en una relación de exterioridad respecto a la sociedad ${ }^{4}$ de la cual forma parte. De este modo, se supone que universidad y sociedad son dos estructuras distintas, entendidas como dos sistemas que pueden interactuar (noción de acoplamiento estructurals).

Lo dicho anteriormente implica que los modos o discursos y acciones que se llevan adelante en la actualidad, bajo la exaltación de lo local, del entorno, plantean una dualización. Es decir, desde esta concepción la universidad estaría por definición alejada de la sociedad a la que pertenece, alejada de su territorio, por lo tanto debería elaborar estrategias de vinculación local, territorial. Solamente así,

[...] reafirmaría su pertenencia al medio y se sentiría parte de él. Esto establecería una gran diferencia, pues la universidad se conec-
3 VILALTA, PALLEJÀ, 2002.

${ }_{4}$ CHAUI, 2003.

5 El acoplamiento estructural puede ser visto como una historia de interacciones recurrentes que conducen a una congruencia estructural entre dos o más sistemas (MATURANA; VARELA ,1998). 
taría a la complejidad de la realidad, en lugar de encerrarse en una cápsula teoricista, vacía y aséptica. La universidad se convierte en parte del medio y esto la hace consciente de las consecuencias de sus acciones [...] (TOSTADO, 2004).

\section{Un rasgo diferencial}

La reformulación del vínculo con lo social se encuentra instalada en una definición de lo local, donde lo local representa un nuevo espacio, una nueva configuración. Lo local /la comunidad/ constituye parte central en al trama discursiva en torno al cual se proyectan las universidades nacionales de reciente formación. El período 1995/200o puede ser considerado como representativo de las tendencias de cambio de las decisiones en la creación de nuevas carreras y de diferentes consideraciones y servicios hacia lo local. Los discursos de presentación se configuran en torno a esta política como un rasgo diferencial, caracterizadas por un intento de acción o de relación hacia o con la comunidad. No obstante, el hecho de referirse a la comunidad, a lo local no indica que su acción esté directamente dirigida a una población marginal.

Tal como plantea De Marinis, (2005) "[...] pese a que en reiteradas ocasiones se le ha extendido a la comunidad su certificado de defunción, ella ha sido recientemente reactivada y reinventada por las racionalidades políticas contemporáneas."

Ejemplos de cómo es recuperado lo local/ lo territorial o la comunidad local, podemos encontrarlos en la Ley de Educación Superior No 24521, Sancionada el 20 de Julio de 1995 y Promulgada el 7 de Agosto de 1995 (Decreto 268/95) bajo la presidencia del Dr. Carlos Menem, En el artículo 56, se encuentra que:

Los estatutos podrán prever la constitución de un Consejo Social, en el que estén representados los distintos sectores e intereses de la comunidad local, con la misión de cooperar con la institución universitaria en su arti- 
culación con el medio en que esta inserta. Podrá igualmente preverse que el Consejo Social éste representado en los órganos colegiados de la institución.

Por otra parte en la Sección 3, en cuanto al sostenimiento y régimen económico-financiero, encontramos en el que artículo 60 plantea:

Las instituciones universitarias nacionales podrán promover la constitución de fundaciones, sociedades u otras formas de asociación civil, destinada a apoyar su labor, a facilitar las relaciones con el medio, a dar respuesta a sus necesidades y a promover las condiciones necesarias para el cumplimiento de sus fines y objetivos. No sólo llama la atención el grado de ambigüedad presente, en cuanto a como se definen las demandas, quien las establece, por medio de que mecanismos, sino también que el último articulo esta relacionado al régimen económico de una institución nacional.

La distinción por lo local, la comunidad local o lo territorial, se encuentra reflejada en los Estatutos de las universidades que fueron creadas en la Argentina en el marco de la nueva ley de Educación Superior. Entre I988 y I995 se crearon I I universidades nacionales. Cinco de ellas nacieron en distintas provincias de la Argentina y otras seis se instalaron en la periferia de la Capital de Buenos Aires (Conurbano). Estas últimas comparten como rasgo común y distintivo una denominación que explicita su pertenencia al Partido o localidad donde se asientan.

A continuación se presentan algunas de las referencias presentes en distintos estatutos de universidades de reciente creación ${ }^{6}$ en cuanto a la mención de la comunidad local, a lo regional.

En las universidades denominadas tradicionales o antiguas (como en el caso de la UBA) encontramos presentes en sus estatutos referencias a objetivos culturales, científicos y profesionales, en función de intereses nacionales e internacionales. En cambio, en las universidades de reciente creación, si bien continúa presente para la mayoría de los casos el carácter universal (en algunas de manera más explícita que en otras) aparece en todos los casos la referencia a lo local, a la comunidad, a lo regional como objetivo privilegiado de acción institucional.
6 Se tomaron como casos de análisis las siguientes universidades publicas argentinas: Universidad Nacional General Sarmiento (UNGS), Universidad Nacional General San Martín (UNSAM), Universidad Nacional de Patagonia Austral (UNPA), y la Universidad Nacional de la Matanza (UNLaM). 
Para el caso de la UNSAM si bien en su estatuto la universidad se define desde una lógica universalista como "[...] una comunidad de estudio, enseñanza, investigación y extensión, comprometida en la búsqueda universal de la verdad [...]", encontramos que también se explicitan objetivos dirigidos a la comunidad tales como: a) extender su acción y sus servicios a la comunidad, con el fin de contribuir a su desarrollo y transformación, estudiando en particular los problemas nacionales y regionales y prestando asistencia científica y técnica al Estado y a la comunidad. Art. 28 de la Ley 2452I- b) en el Art. 5 se plantea que para servir a las necesidades de la comunidad la universidad mantendrá una permanente vinculación con el sistema educativo, con el gobierno nacional, los gobiernos provinciales, los municipios de su zona de influencia, las instituciones intermedias de la región y las fuerzas de la producción y el trabajo de modo de conocer sus necesidades y recibir su aporte. c) una relación continua con organizaciones profesionales, científicas, técnicas, culturales y religiosas de índole nacional o internacional, para enriquecer los planes y programas de estudio e investigación y la transferencia de conocimientos y tecnologías. d) la UNSAM define como su misión fundamental "[...] brindar formación de calidad, integrando enseñanza, investigación y experiencia, y realizar transferencia de conocimientos y asistencia al sector público y privado, para mejorar las condiciones sociales, económicas y culturales de la comunidad [...]"

Para el caso de la UNPA, constituida a partir de la Universidad Federal de la Patagonia Austra,l creada por el Tratado celebrado entre las Provincias de Santa Cruz y Tierra del Fuego el 6 de diciembre de I990, aprobado por Ley de la Provincia de Santa Cruz No22 I2, y creada, sobre esta base, por ley del Congreso Nacional No 24.446, encontramos presente en su estatuto que la universidad tiene por objeto "promover acciones tendientes al desarrollo socio-económico regional y nacional y a la preservación del medio ambiente", además de "facilitar y coordinar las acciones sobre Educación y Ciencia y Tecnología.” La UNPA tiene por principal objeti- 
vo responder a las demandas regionales de educación superior, no sólo de formación profesional sino fundamentalmente en la producción de conocimientos científicos y tecnológicos 7 .

Un rasgo distintivo presente en las declaraciones formales de la UNLaM se encuentra en el Art. 5 se su de su estatuto universitario. Allí se menciona entre las finalidades de universidad "[...] servir a las necesidades de la 'comunidad': de sus 'empresas', de sus establecimientos".

Por último, en la UNGS encontramos en el Art. I su estatuto universitario la necesidad de

[...] participar e incidir en los esfuerzos que la comunidad realiza para su pleno desarrollo a través de la pertinencia de la investigación, la formación adecuada, la efectividad de los servicios y la flexibilidad en las estructuras universitarias. La acción local y el pensamiento universal son complementarios.

Este recorrido sucinto por los estatutos universitarios permite entrever para el caso de estas denominadas nuevas universidades una fuerte incorporación en sus dinámicas institucionales de la comunidad donde se encuentran emplazadas y una complementariedad en su propuesta institucional entre la noción de comunidad y Nación. Según Renato Ortiz (2002, p. 58) al hablar de local, nacional y global establecemos un ordenamiento entre niveles espaciales diferenciados, lo que nos lleva, necesariamente, a pensar las relaciones entre ellos. De esta manera se enfatiza la intersección, interacción y mutua determinación de los niveles global, nacional y local.

Sin embargo, la referencia a lo local presente tanto en el discurso fundacional como en sus respectivos estatutos adquiere posteriormente diversos sentidos en las dinámicas de proyectos institucionales, y solo un análisis en profundidad permite entrever que es lo que se considera por "vínculo con el entorno" en términos operacionales y en dónde se instala el énfasis en la rela-
7 Cabe destacar, "[...] que al momento de su creación como universidad nacional esta institución ya contaba con experiencias de vinculación con el Gobierno Provincial y algunas empresas de la región, lo cual ha marcado un perfil que se intenta seguir manteniendo. En la actualidad se pretende profundizar estas vinculaciones acentuando la atención hacia demandas "del medio" en el que está inserta y, además, atendiendo más fuertemente a la generación de nuevas fuentes de financiamiento que posibiliten su sostenimiento y desarrollo." (GALARETTO; AMELIO; ROMERO, 2007).

$8 \mathrm{El}$ grifo es nuestro. 
9 STURNIOLO. Inflexiones en el discurso académico: la universidad y su articulación local. (en prensa).

Io En las entrevistas realizadas notamos que estos términos son utilizados como sinónimos al referirse a la vinculación de la universidad en el lugar de su emplazamiento. ción de la universidad con los diferentes actores sociales que componen la comunidad: Estado- Empresas (multinacionales y/o locales, pymes), Gobierno local, otras instituciones?.

Por ultimo, es de destacar que las implicaciones de la relación entre la universidad y el territorio/ la comunidad/ o lo local ${ }^{\text {Io }}$ están vinculadas a una nueva y necesaria redefinición de la función social de las universidades, fundamentalmente a modo de moderación de los cambios producidos por los procesos de globalización y exaltación de la vida local. En este caso el discurso de lo local actuaría como una especie de sutura en la fractura del vínculo universidad- sociedad, a partir de lo cual lo fragmentado se uniría otorgando un sentido a la acción.

\section{Consideraciones finales}

La instalación de "lo local", no se halla exclusivamente en un plano discursivo gramatical, sino que surge de un régimen de signos, de una organización de poder. Es decir, no hay nada aséptico, neutral, en esta forma de definir de nombrar una relación, una misión institucional, una propuesta de formación. La reformulación del vínculo con lo social se encuentra instalada en una definición de lo local, donde lo local representa un nuevo espacio, una nueva configuración. Si bien no podemos aún hablar de un viraje del modelo universitario, encontramos una clara tendencia de diferenciación asociada al momento de su creación y a la tradición de las universidades indagadas. Las políticas implementadas por estas nuevas instituciones, si bien no operan cambios rotundos en el esquema universitario se puede ver cómo incorporan y, a la vez, introducen nuevas tendencias donde lo local intenta posicionarse como una nueva identidad, que como diría Foucault (I999) "[...] identidad, bien débil [...] el plural la habita, numerosas almas se pelean en ella; los sistemas se entrecruzan y se dominan los unos a los otros." 


\section{The UNIVERSITY IN FRONT THE LOCAL / TERRITORIAL NEEDS AND DEMANDS}

The intention of this article is to make questionable the resurgence of the demands for an attention to the local/ territorial/ or to the local community that is performed at the university, demands which in its turn are commensurate to the processes of social change, and in an underlying a discussion about the redefinition of the social function of universities. The period $1995 / 2000$ can be considered as representative of the trends of decisions changing in the creation of new careers and of different considerations and services toward the local. The speech presentations are configured around this policy as a differential feature, characterized by action intention or relationship toward or with the community. Arises in this context a demand toward a linkage with the environment, for the attention to the local, by the need for territorial responses. The local is one of the discursive forms present in the academic ambit in the last decade. The local as a category refer us to the university- society link, widely discussed redefined and necessarily questioned.

KeY wORDs: Demands. Social Function. University.

\section{Referencias}

ARMELLA, J.; KANTAROVICH, G.; STURNIOLO, S.; RODRIGO, I. Crisis universitaria: hacia la búsqueda de nuevos sentidos. En: Perspectivas criticas desde el siglo XXI sobre la educación en Argentina y Canadá. Estudios comparados y lecciones de las experiencias. Buenos Aires: Editado por Llomovatte; Naidorf, 2006.

BOISER, S. Una (re)visión heterodoxa del desarrollo (territorial): un imperativo categórico. En: ROFMAN, A. Universidad y Desarrollo Local. Aprendizajes y desafios. Buenos Aires: Prometeo, 2005.

CHAUI, M. Sociedade, universidade e Estado: autonomia, dependência e compromisso social Seminário: Universidade: Por que e como reformar? MEC/SESu: 6 e 7 de agosto 2003. Disponible en: $<$ http://www.firgoa.usc.es/drupa/filestore2/dowload/19336/MarilenaChaui.pdf>.

DELEUZE, G. Foucault. Argentina: Paidós, 2008.

DERRIDA, J. La Universidad sin condición. Madrid: Mínima Trotta, 2002. 
FERRER,D.; BRUNO, J.; MARÍ, A., 2002. La contribución de las universidades al desarrollo sostenible a través de la formación. El caso de la universidad politécnica de Cataluña. En: VILALTA, J.; PALLEJÀ, E. (Ed.). Universidades y desarrollo .territorial en la sociedad del conocimiento . Barcelona: Diputació Barcelona y Universitat Politècnica de Catalunya, 2002. v. II.

FOUCAULT, M. Elorden del discurso . Barcelona: Fábula Tusques, I999.

GRINBERG, M. Educación y poder en el siglo XXI. Gubernamentalidady pedagogía en las sociedades del conocimiento. Argentina: Miño y Dávila, 2008.

LEY 24.082. Universidades Nacionales-Universidad Nacional de GeneralSarmiento Ley de Educación Superior 24.521.

NAISHTAT, F. Proyecto TS28: El giro sistémico en la Educación Superior y el sujeto universitario. Disponible en: <http://simel.edu.ar/invest/res_ubacyt.pdf $>$.

NUEVAS UNIVERSIDADES. Asuntos Académicos. Acuerdo Plenario, No 325. Córdoba, I9 de abril de 1999 .

ORTIZ, R. Otro territorio. Ensayos sobre el mundo contemporáneo. Bernal. Buenos Aires: Universidad Nacional de Quilmes, 2002.

RESOLUCIÓN CONSEJO SUPERIOR No 285/oo. Expediente N4238/00. San Miguel, 24 de febrero de 2000 .

TOSTADO, G. A. Complejidad, transdisciplina y redes: hacia la construcción colectiva de una nueva universidad. Revista Académica Polis, 2004. Disponível em: <http://www.lafogata.org/ opiniones/aiz_complej.htm>.

UNIVERSIDAD NACIONAL DE GRAL. SARMIENTO, Estatuto Universitario, 1998. UNIVERSIDAD NACIONAL DE PATAGONIA AUSTRAL, Estatuto Universitario, UNPAUACO, I999.

UNIVERSIDAD NACIONAL GENERAL SAN MARTÍN, Estatuto Universitario, I998.

VILALTA, J.; PALLEJÀ, E. Universidades y desarrollo territorial en la sociedad del conocimiento. v. II Barcelona: Diputació Barcelona y Universitat Politècnica de Catalunya, 2002.

Recebido em Io set. 2009 / Aprovado em 2I dez. 2009

Para referenciar este texto

STURNIOLO, S. A. La universidad frente a las necesidades y demandas locales / territoriales. EccoS, São Paulo, v. I I, n. 2, p. 457-466, jul./dez. 2009. 